International Journal of Distributed and Parallel Systems (IJDPS) Vol.3, No.6, November 2012

\title{
ENHANCEMENT OF TCP PERFORMANCE WITH THE HELP OF SNOOP PROTOCOL IN WIRED CUM WIRELESS NETWORK
}

\author{
Maninder Kaur ${ }^{1}$ and Parminder Singh ${ }^{2}$ \\ ${ }^{1}$ Research Scholar, Department of Information Technology, CEC Landran \\ maninder.parmareymail.com \\ 2 Assistant Professor, Department of Information Technology, CEC Landran \\ singh.parminder06@gmail.com
}

\begin{abstract}
The TCP networks have been the only communication for wired media that followed IEEE 802.3 networks. This paper attempts to provide a comprehensive overview of this Link Layer approach i.e. Snoop protocol that improving throughput of TCP in wireless networks. It first explains the role of Snoop protocol that overcomes the limitations of TCP protocols in wireless media. We investigated the application of this protocol in wireless networks and observed that a single hop in the wireless network experienced large variations in round trip time in a very short period. Without changes to the Snoop protocol to accommodate these dramatic RTT variations, Snoop was performing badly compared with regular TCP even when there were no packet losses or errors. The main cause for this is premature retransmissions performed by Snoop. We have modified the Snoop protocol to avoid these unnecessary retransmissions by having a higher local retransmission timeout. The results showed us that Snoop benefits from this approach which has made a significant performance improvement over regular TCP in multihop wired cum wireless networks.
\end{abstract}

\section{KEYWORDS}

Snoop, RTT, TCP, MultihoP

\section{INTRODUCTION}

TCP was designed to run over any packet-switching wired networks. TCP provides reliable service without overloading the network. Popularity of TCP leads the network engineers to apply TCP in wireless technology as well. In addition to many services such as flow control, congestion management and reliability, TCP attempts to provide both an efficient utilization and a fair share of network resources. There are various reasons due to which TCP performance is not good in wireless environment that are random losses that come from high bit-error rate, low and variable bandwidth, and frequent disconnections.

The reason is not the retransmission of the lost packets, but the congestion control (CC) algorithms that are forced to respond to losses. Almost always, TCP assumes that a lost packet signals network congestion. TCP is equipped with good congestion control mechanism. A congestion window (cwnd) is maintained which gives information about current estimate of the available capacity of the network. At any moment, sender is allowed to send no more than cwnd number of segments without any acknowledgement from the receiver. As soon as the loss is indicated by a timeout, tc--p enters into a slow-start phase. When the loss is detected by the sender a receipt of three duplicate acknowledgements get from the destination, then the system goes into fast recovery. .The result is the reduction of the congestion window size. On the other hand, whenever a packet loss is due to a transmission error, any contraction of the window is inappropriate. The outcome is decreased throughput, poor utilization of the network resources, and marginal performance of the applications. [2].Congestion in wireless networks is slightly different from that of wired networks.We group them into two types: Type A and Type B.In

DOI : $10.5121 /$ ijdps.2012.3612 
International Journal of Distributed and Parallel Systems (IJDPS) Vol.3, No.6, November 2012

Type A: in a particular area, many motes within range of one another attempt to transmit simultaneously, resulting in type II losses and thereby reducing throughput of all motes in the area and In Type B: within a particular mote, the queue, or buffer used to hold packets to be transmitted, overflows. This is the conventional definition of congestion, widely used in wired networks.[10]. TCP assumes each packet loss as a sign of congestion and sets ss threshold to half of the cwnd and sender enters again into slow start phase. Fast retransmit is a technique used for recovery when packet loss is detected before timeout [1].By setting the maximum congestion window size as gives maximum throughput in most, though not all cases. Changing the window size has no effect on throughput for 1-hop flows, while it does affect the throughput as the number of hops increases [3].

\subsection{Snoop Protocol}

Snoop is a TCP-aware link layer protocol. It is designed to improve the performance of TCP in the wired-cum-wireless networks. The Snoop Protocol [4] was designed to solve the burst/intermittent packet loss due to high bit error rates and short temporary disconnections experienced by TCP in wireless link. The main idea of the protocol is to cache packets at the base station and perform local retransmissions across the wireless link.[5]The concept is based on a snoop agent or snoop module that is added to the routing code on the base station to monitor each single packet passing through the connection. The snoop module maintains a cache to temporarily store packets sent from the wired network to mobile hosts that have not been yet acknowledged by the mobile host. For transfer of data from a fixed host to a mobile host, modifications are made only to the routing code at the base station. These modifications include caching unacknowledged TCP data and performing local retransmissions based on a few policies dealing with acknowledgments (from the mobile host) and timeouts. This results in a significant improvement of connection performance, without sacrificing the end-to-end semantics of TCP, modifying host TCP code in the fixed network, or re-linking existing applications[5].The base station routing code is modified by adding a module, called the snoop, that monitors every packet passing through the connection in either direction. When a new packet sent from a fixed host arrives at the base station, the Snoop module will add it to the cache. Then it forwards the packet to the routing code that routes it to the appropriate mobile host. The snoop module relies on a cache of un-acknowledged packets to improve end to end TCP semantics. Typically, the size of this cache is proportional to the TCP window size.[9]

\section{BACKGROUND WORK}

TCP was originally designed primarily for wired networks. In a wired network, random bit error rate, a characteristic usually more pronounced in the wireless network, is negligible, and congestion is the main cause of packet loss. The emerging wireless applications, especially high-speed multimedia services and the advent of wireless IP communications carried by the Internet, call for calibration and sophisticated enhancement or modifications of this protocol suite for improved performance. Based on the assumption that packet losses are signals of network congestion, the additive increase multiplicative decrease congestion control of the standard TCP protocol reaches the steady state, which reflects the protocol's efficiency in terms of throughput and link utilization. However, this assumption does not hold when the end-to-end path also includes wireless links. Factors such as high BER, unstable channel characteristics, and user mobility may all contribute to packet losses. Many studies have shown that the unmodified standard TCP performs poorly in a wireless environment due to its inability to distinguish packet losses caused by network congestion from those attributed to transmission errors.[7]In heterogeneous networks, to explicitly differentiate the cause of packet loss becomes the primary goal of TCP design. Such efforts aim to find an explicit way to inform the sender of 
the cause of packet dropping, be it congestion or random errors. Therefore, the sender is able to make appropriate decisions on how to adjust the congestion window. The standard Reno scheme halves its window size when experiencing a packet loss regardless of the cause. If the loss is due to network congestion, this behavior alleviates network congestion. However, it would degrade the performance for random loss. Explicit Congestion Notification (ECN), Snoop protocol and their combination can be used to improve the performance of TCP in WiMax network, ECN will help in congestion control and SNOOP will retransmit the packets that are lost from nodes in between, saving nearly half the retransmission time and avoiding the decreasing in transmission speed. The performance of these protocols is investigated over WiMax wireless scenario which improves the various parameters of TCP such as throughput. The results of the same can be demonstrated on NS2 simulator [4].Some researchers have attempted to provide solutions at the Data Link Layer (LL), thereby attempting to hide the "deficiencies" of the wireless channel from TCP. Others have introduced modifications to TCP so that it performs better under the new conditions. Last but not least, there are a number of proposals for new transport protocols, optimized for wireless networks. Some proposals are TCP-aware LL protocols, TCP-unaware LL protocols, Split connection and many more [8].

\section{SIMULATION STUDY}

NS2 is a simulation tool widely used by people in the network research community. NS2 provides substantial support for TCP, routing and other protocols over different mediums including wired and wireless networks [6]. The Snoop protocol is one among the NS2's supported protocols allowing one to simulate and study the protocol behaviour. But the current implementation of Snoop in NS2 does not allow simulating a desirable topology where the Snoop protocol is highly applicable. The Snoop protocol was originally applied in a wired-cumwireless network. For a typical wired- cum-wireless link it is assumed that the wired link is more reliable compared with the wireless link. The NS2 Snoop implementation allows a wired erroneous network simulation to simulate the effect of the Snoop protocol.

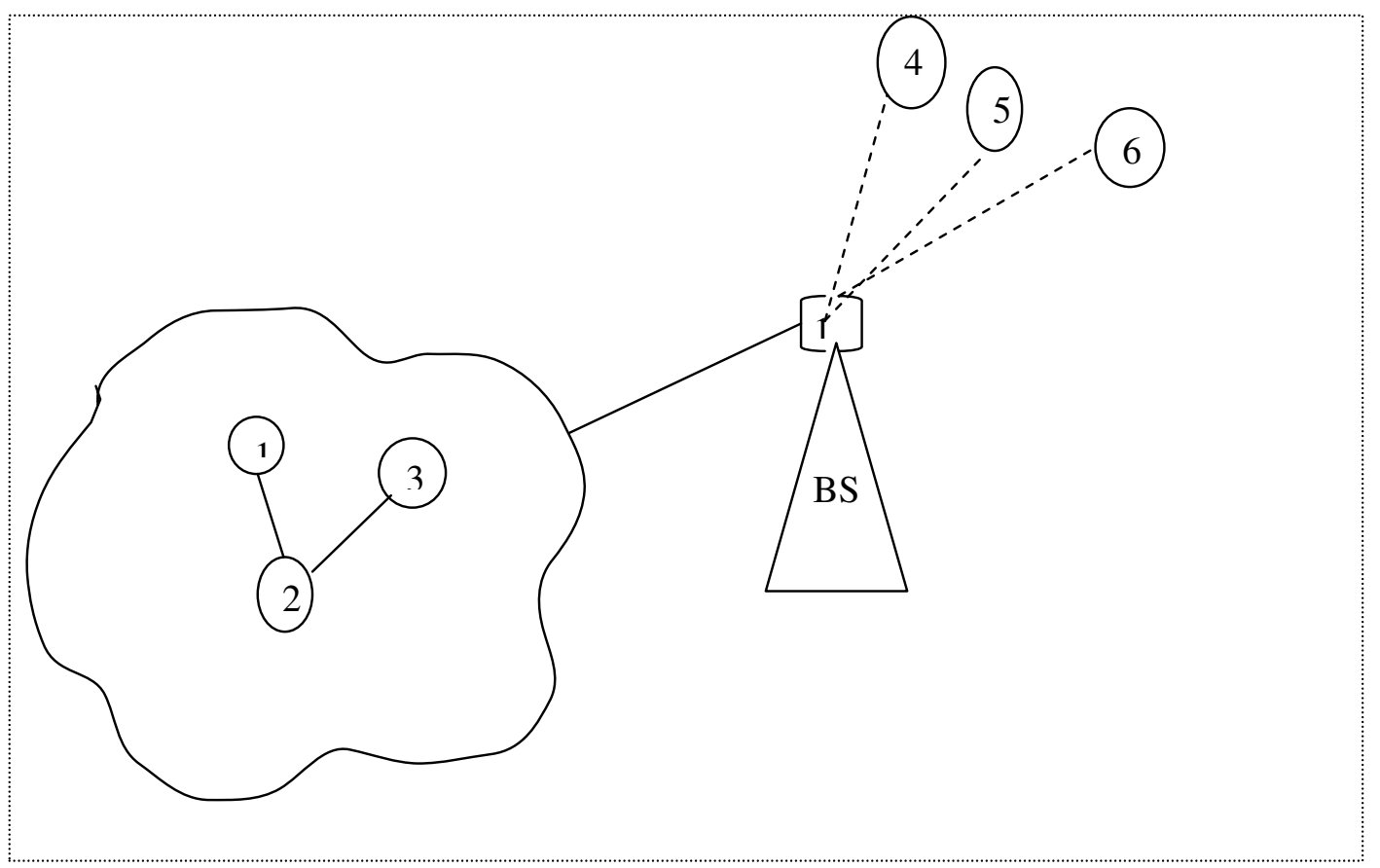

Fig 1: Wired cum Wireless Scenario. 
In above scenario, we used NS2 to perform the simulation of a wired cum wireless Scenario that consist of 6 nodes. The three nodes i.e. Nodes1, 2, 3 are wired network and node 4,5,6 are in wireless fashion. The base station lies between wired and wireless network. We applied Snoop protocol on this base station and all nodes that are part of network so that it monitors the performance of TCP in both environments. The parameters used were two way propagation model, 802.11 as the MAC, Omni directional antenna and drop tail interface queue with length of 50 for every node. We used Destination-Sequenced Distance-Vector Routing (DSDV) as the routing algorithm.

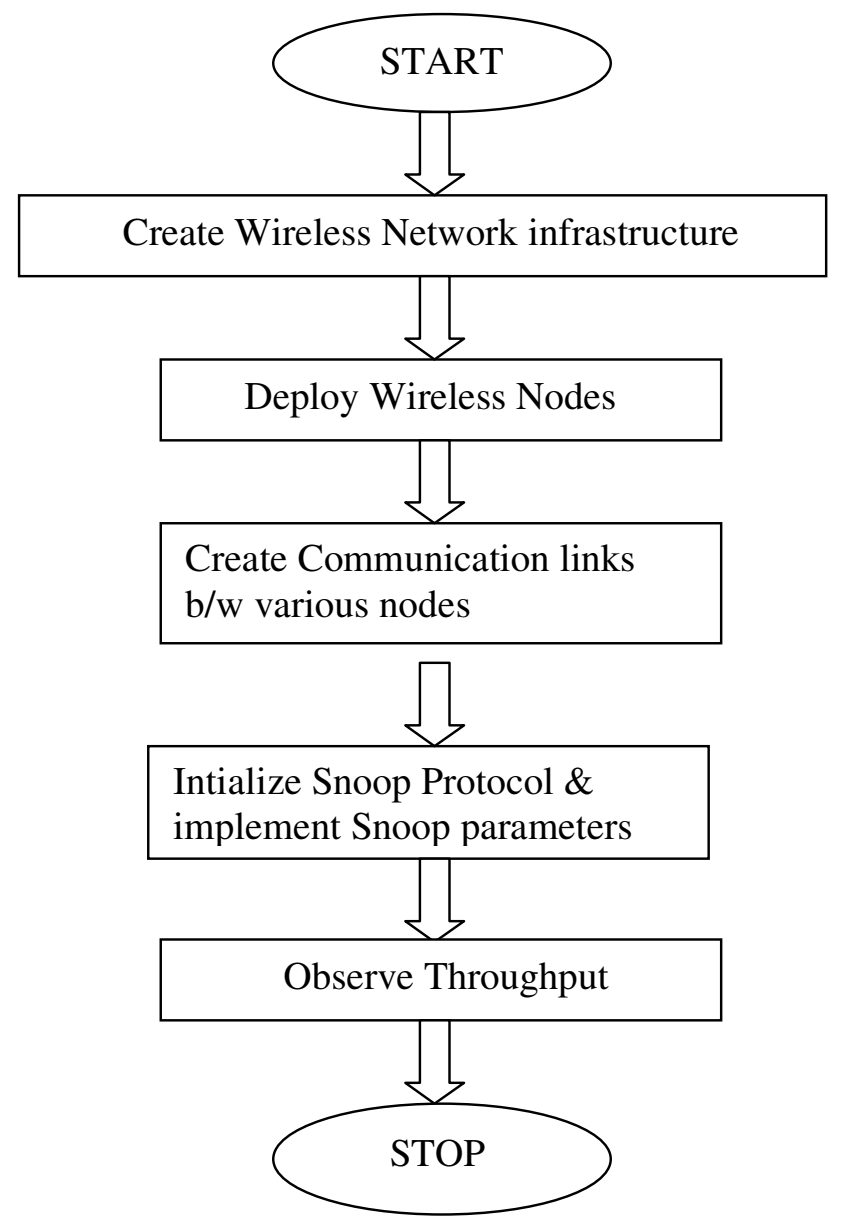

Fig. 2 Simulation setup flow diagram 
International Journal of Distributed and Parallel Systems (IJDPS) Vol.3, No.6, November 2012

\section{SIMULATION RESULTS}

This section discusses simulation results performed on TCP regular flow control and TCP with SNOOP protocols.

\begin{tabular}{|c|c|}
\hline Simulator & NS-2.35 \\
\hline Channel type & Wireless phy \\
\hline Transmission Rate & $10 \mathrm{mbps}$ \\
\hline Mobility Model & Two ray ground model \\
\hline Packet size & 1024 bytes \\
\hline Buffer size & 20 bytes \\
\hline Simulation Time & $200 \mathrm{sec}$ \\
\hline Antenna model & Omni directional \\
\hline No.of mobile nodes & 3 \\
\hline Routing protocol & DSDV \\
\hline Network size & 5 \\
\hline Queue size & 50 \\
\hline
\end{tabular}

Table No.1 


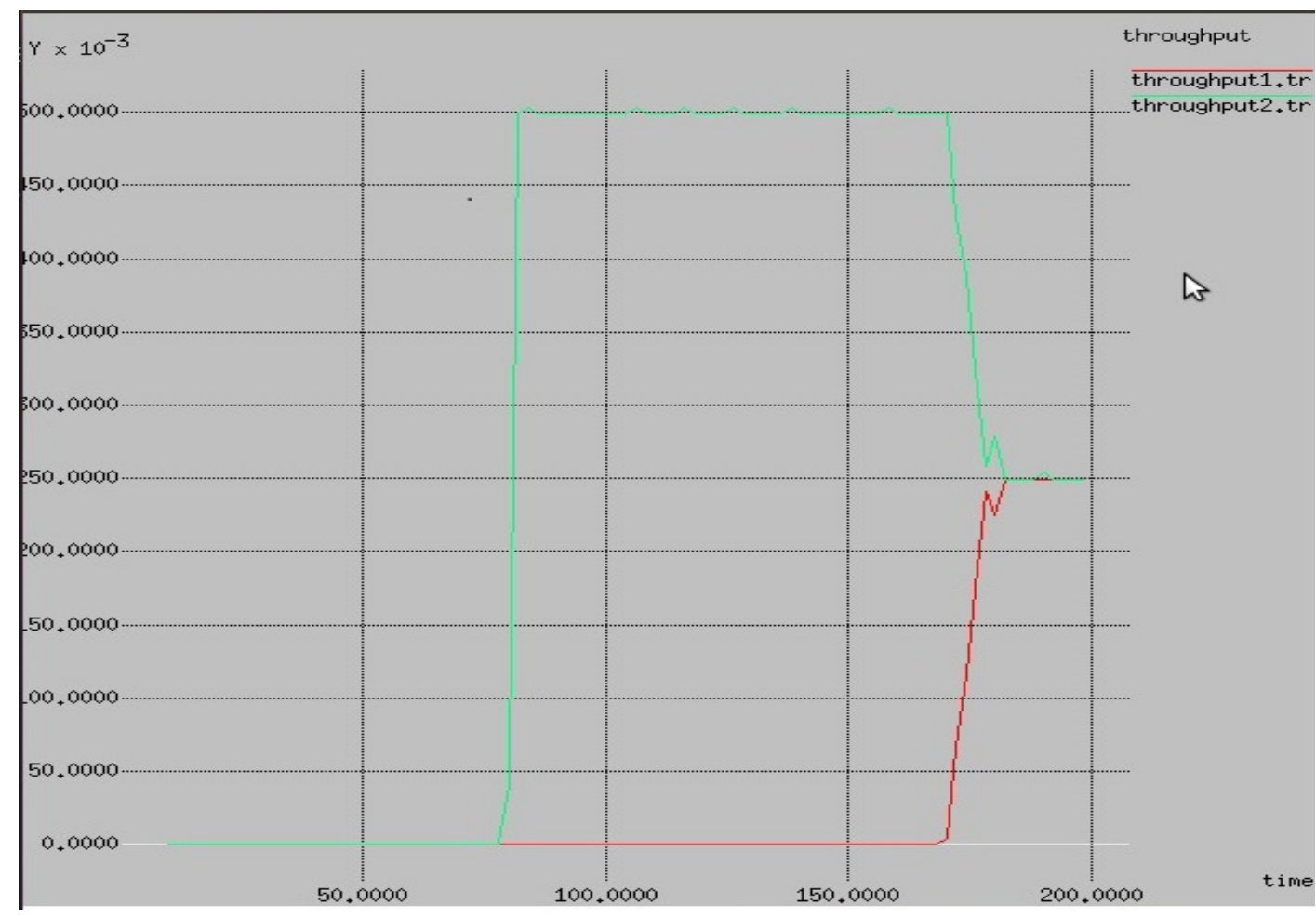

Fig. 3: Throughput of TCP regular flow vs TCP for snoop

The first graph in figure 3 compares the throughput between tcp for regular flow and tcp for snoop. It is apparent from the graph that the queuing delay plays an important role in the simulation. Here queuing delay is the duration between a packet leaving the agent and being sent on the medium.

1) It is well illustrated from the graph that the green line indicates the snoop behavior and red line indicates the regular flow. Initially, due to effect of delay the both flow remain constant for certain interval of time after the throughput of regular flow increases steady and the throughput of snoop flow decreases. After that a little variations in their throughput, the regular flow bent down slightly and snoop flow bent up due to the effect of snoop round trip and queuing delay. After certain interval of time they force to remain steady which shows the end of simulation but slight increase in the variations of normal flow of tcp.

2) It is well illustrated from the graph that the effect of snoop behavior increases at certain point in compare to regular flow which shows the pyramid like bent up the throughput green line and pyramid like bent down of red line in normal flow due to by improving the RTT (Re transmission Time out ). RTT is the amount of queuing delay between nodes and the transmission time of TCP packet and ACK packet. But, it is well illustrated from the graph that the RTT is not proper in this simulation due to the congested network, so there will be various variations in the nature of throughput of regular and snoop flow. In this simulation, the flow of throughput is not proper and steady. There is sudden up and down variations in both flows. 


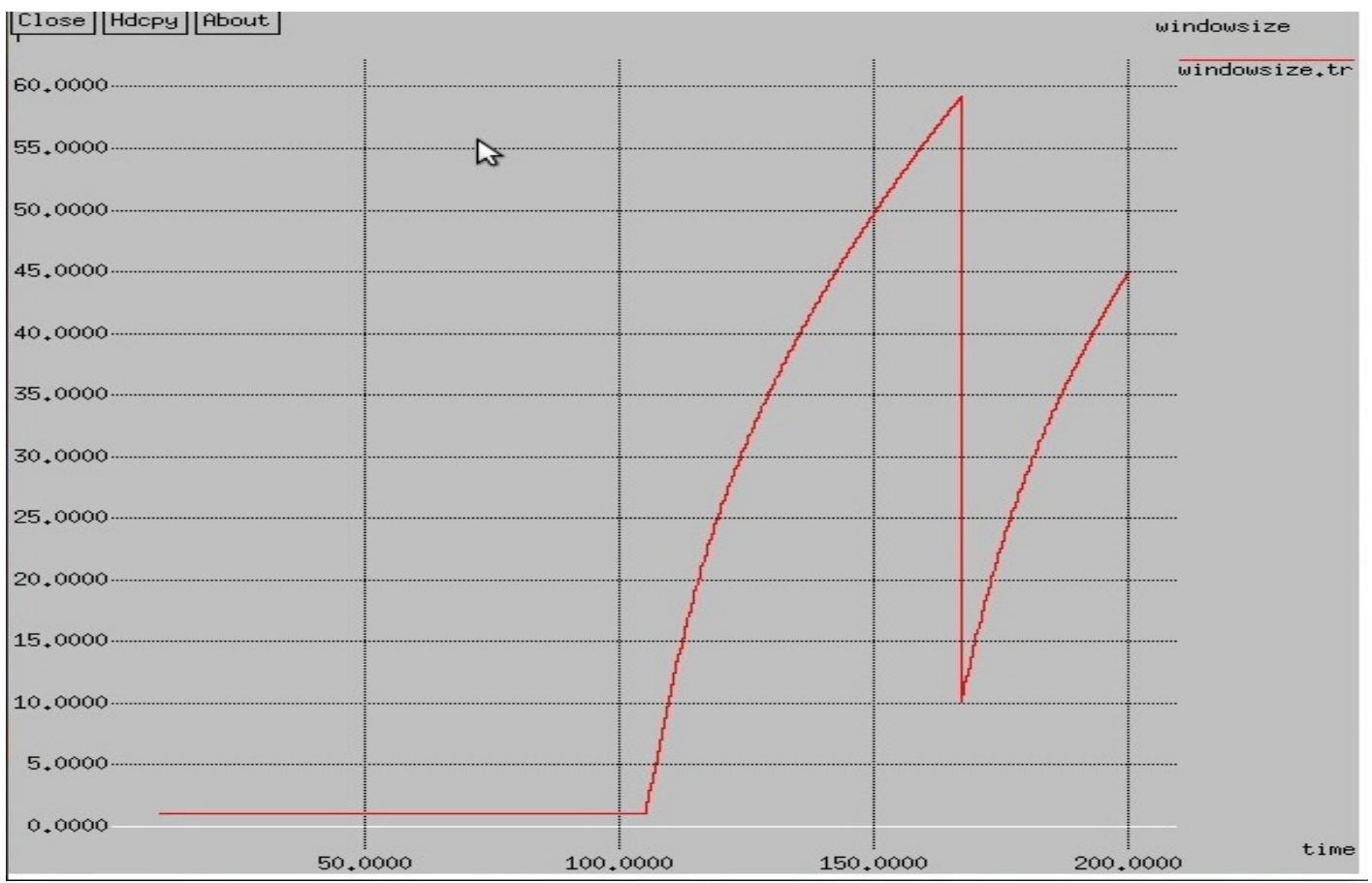

Figure 4: TCP window size variation

Figure 4 graph illustrates the variation in tcp window size with respect to time. At initially, it becomes constant and steady up to 100 seconds but after it there is sharp increase in the behavior of window size due to various variations of throughput and at particular time it constantly increase and further increase in their behavior. It is due to various variation nature of RTT and queuing delay which always try to improve their nature in the whole simulation which is clearly apparent from the above graph. 


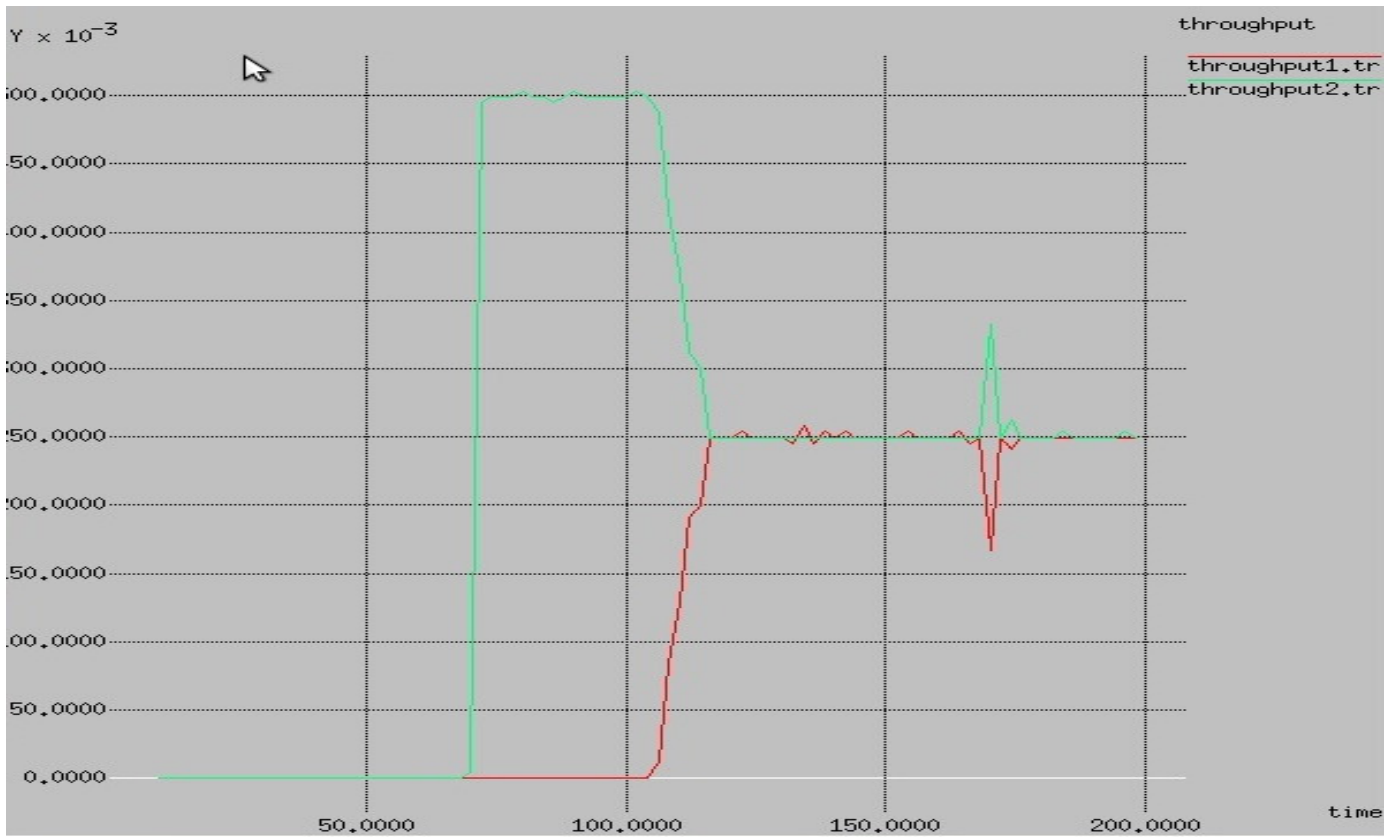

Fig. 5: Improved SNOOP flow

The above graph in figure 5 shows the improved nature of snoop flow with compare to regular flow with effect of retransmission in congested network. The simulation tries to implement higher retransmission timer which further helps to boost the snoop performance as compared to original approach. It is clearly apparent from the graph that the nature of snoop indicated by green line have less variations when it is very closer to regular and it is smooth straight line and no dip in the graph indicates that when the retransmission timeout is set to very low the snoop flow performs better performance at retransmission. On the other hand, the performance of snoop throughput is quiet high as compare to normal flow which is shown in the graph at time 170 s which indicates that the behavior of snoop is quiet improved in the simulation due to improved in nature of RTT and queuing delay. The above simulation is quiet suitable which shows improved nature of snoop in the whole environment.

\section{CONCLUSION}

In this paper, the snoop protocol has been proposed to improve TCP throughput in wired cum wireless network. As, TCP suffers from a various problems in wireless networks. But our simulation environment is created in such a way so that snoop is applied on every node in wired as well wireless environment. Firstly by using snoop, the performance of TCP decreased due to premature retransmissions that occur only because of low retransmission time out. But retransmission timeout is set high; it shows little improvement in results. We applied snoop at every node and proved that snoop helps TCP to get better results. our simulation show that the a wireless network is more complicated than wired networks with a higher error rate. 


\section{FUTURE WORK}

There are other possible scenarios in which the network either can be heterogeneous or homogeneous or network can be wireless or wired. We have to understand the impact of its semantics with respect to throughput. We suggest for future the impact of packet loss due to multiple reasons that need further investigation. Especially, when it is under same adversity or an attack. Because we do not know how snoop will behave when it comes under attack and same security is compromised. Therefore, this aspect is also need to take care for further future scope.

\section{REFERENCES}

[1] Md. Ehtesamul Haque and Md. Humayun "TCP Congestion control in Heterogeneous Network "Kabir Department of Computer Science and Engineering Bangladesh University of Engineering and Technology, Dhaka 1000, Bangladesh.

[2] Christina Parsa and J.J. Garcia-Luna"Improving TCP Congestion Control over Internets with Heterogeneous Transmission Media “Aceves Computer Engineering Department.

[3] Dimitrios Koutsonikolas, Jagadeesh Dyaberi et.allOn TCP Throughput and Window Size in Multihop Wireless Network Testbed,Center for Wireless Systems and Applications,Purdue University

[4] Mr. Manish, D.Chawhan, Dr Avichal R.Kapur "Performance Enhancement of TCP Using ECN and Snoop Protocol for Wi-Max Network" Shri Ramdeobaba Kamla Nehru College of Engg, International Journal of Computer Applications.

[5] Ashish Natani, Jagannadha, Jakilinki,et.all "TCP for Wireless Networks" Computer Science Program,University of Texas at Dallas, Richardson, November 12, 2001.

[6] Prasad Nambiar, et.all "Snoop Behaviour in Multihop Wireless Networks "School of Computer Science University of Hertfordshire Hatfield, Hertfordshire,2010.

[7] YE TIAN, KAI XU "TCP in Wireless Environments: Problems and Solutions" IEEE Radio Communications , March2005.

[8] Kostas Pentikousis "TCP in wired-cum-wireless environments" Department of Computer Science,State University of New York ,2002

[9] Hari Balkrishan, Srinivasan and Randy H.Katz"Improving Reliable Transport and Handoff Performance in Celular Wireless Networks"Electrical Engineering and computer science university of California, Barkeley,Nov. 2004.

[10] Cheng Tien Ee, Ruzena Bajscy Congestion Control and Fairness for Many-to-One Ruting in Sensor Networks "Electrical Engineering and Computer Science University of California, Berkeley, November 2004. 